\title{
Quantum enhanced optomechanical magnetometry
}

Li, Bei-Bei; Bilek, Jan; Hoff, Ulrich Busk; Madsen, Lars S.; Forstner, Stefan; Prakash, Varun; Schäfermeier, Clemens; Gehring, Tobias; Bowen, Warwick P.; Andersen, Ulrik Lund

\section{Published in:}

Optica

Link to article, DOI:

10.1364/OPTICA.5.000850

Publication date:

2018

Document Version

Publisher's PDF, also known as Version of record

Link back to DTU Orbit

Citation (APA):

Li, B-B., Bilek, J., Hoff, U. B., Madsen, L. S., Forstner, S., Prakash, V., Schäfermeier, C., Gehring, T., Bowen, W. P., \& Andersen, U. L. (2018). Quantum enhanced optomechanical magnetometry. Optica, 5(7), [850]. https://doi.org/10.1364/OPTICA.5.000850

\section{General rights}

Copyright and moral rights for the publications made accessible in the public portal are retained by the authors and/or other copyright owners and it is a condition of accessing publications that users recognise and abide by the legal requirements associated with these rights.

- Users may download and print one copy of any publication from the public portal for the purpose of private study or research.

- You may not further distribute the material or use it for any profit-making activity or commercial gain

- You may freely distribute the URL identifying the publication in the public portal 


\title{
Quantum enhanced optomechanical magnetometry
}

\author{
Bei-Bei Li, ${ }^{1,2}$ (i) Jan Bílek, ${ }^{3}$ Ulrich B. Hoff, ${ }^{3}$ Lars S. Madsen, ${ }^{1}$ Stefan Forstner, ${ }^{1}$ Varun Prakash, ${ }^{1}$ \\ Clemens Schäfermeier, ${ }^{3}$ Tobias Gehring, ${ }^{3}$ Warwick P. Bowen, ${ }^{1, *}$ and Ulrik L. Andersen ${ }^{3}$ \\ ${ }^{1}$ School of Mathematics and Physics, The University of Queensland, St Lucia, QLD 4072, Australia \\ ${ }^{2}$ Qian Xuesen Laboratory of Space Technology, Beijing 100094, China \\ ${ }^{3}$ Department of Physics, Technical University of Denmark, Fysikvej 309, 2800 Kgs. Lyngby, Denmark \\ *Corresponding author: w.bowen@uq.edu.au
}

Received 30 April 2018; revised 21 June 2018; accepted 22 June 2018 (Doc. ID 330447); published 12 July 2018

\begin{abstract}
The resonant enhancement of both mechanical and optical response in microcavity optomechanical devices allows exquisitely sensitive measurements of stimuli, such as acceleration, mass, and magnetic fields. In this work, we show that quantum correlated light can improve the performance of such sensors, increasing both their sensitivity and their bandwidth. Specifically, we develop a silicon-chip-based cavity optomechanical magnetometer that incorporates phase squeezed light to suppress optical shot noise. At frequencies where shot noise is the dominant noise source, this allows a $20 \%$ improvement in magnetic field sensitivity. Furthermore, squeezed light broadens the range of frequencies at which thermal noise dominates, which has the effect of increasing the overall sensor bandwidth by $50 \%$. These proof-of-principle results open the door to apply quantum correlated light more broadly in chip-scale sensors and devices. () 2018 Optical Society of America under the terms of the OSA Open Access Publishing Agreement
\end{abstract}

OCIS codes: (120.4880) Optomechanics; (270.6570) Squeezed states; (140.3945) Microcavities; (140.4780) Optical resonators; (280.4788) Optical sensing and sensors; (130.3120) Integrated optics devices.

https://doi.org/10.1364/OPTICA.5.000850

\section{INTRODUCTION}

Cavity optomechanics [1-3] has attracted increasing research interest for both fundamental studies and practical applications. Strong radiation pressure coupling between high quality mechanical and optical resonances has enabled the demonstration of a range of interesting quantum behaviors, such as ground state cooling of macroscopic mechanical oscillators [4-7], quantum squeezing of mechanical motion [8-11], and the production of squeezed light $[12,13]$, while the combination of resonance enhanced mechanical and optical response [14] has enabled precision sensors [15] ranging from kilometer-sized laser interferometer gravitational wave detectors $[16,17]$ to micro/nanoscale siliconchip-based force [18], mass [19], acceleration [20,21], and magnetic field [22-25] sensors.

The precision of cavity optomechanical sensors is generally constrained by three fundamental noise sources: thermal noise from the environment, shot noise from the photon number fluctuations of the light used to probe the system, and quantum backaction noise arising from the radiation pressure of the probe light. The noise floor can be engineered using quantum correlated light. For instance, squeezed light [26-28] allows the shot noise to be suppressed [29], thereby improving the sensitivity if the shot noise is dominant. Squeezed light has been used, for example, to improve the precision of gravitational wave interferometry in both the Laser Interferometer Gravitational-Wave Observatory (LIGO) and the GEO600 [30-32], and it has also been used in nanoscale measurements of biological systems [33], laser beam positioning [34,35], and magnetic field measurement using atomic magnetometers $[36,37]$. In cavity optomechanics, it has been used to enhance measurements of thermal noise [38], improve both feedback [39] and sideband cooling [40], and study the backaction from the radiation pressure force [41]. However, it has not previously been used to improve cavity optomechanical sensors of external stimuli. Here, we demonstrate the first application of squeezed light in such a sensor, specifically, in a cavity optomechanical magnetometer $[22,23]$. At frequencies where shot noise is dominant, squeezed light suppresses the noise floor, improving the magnetic field sensitivity. Moreover, by increasing the range of frequencies over which thermal noise is dominant, the sensor bandwidth is also increased. A squeezed light enhanced sensor bandwidth [42] is of importance in applications that need good sensitivity in a broadband range, e.g., in magnetic resonance imaging.

\section{THEORETICAL ANALYSIS}

Figure 1(a) shows a conceptual schematic of a cavity optomechanical magnetometer, comprised of an optical cavity, coupled to a mechanical oscillator. The mechanical oscillator is driven by a force $F_{\mathrm{B}}$ induced by a magnetic field via the magnetostrictive effect [22] along with thermal and backaction noise forces. The mechanical motion of the oscillator changes the cavity length and, thus, the optical resonance. This modulates the phase of an injected squeezed probe field and can therefore be read out via an optical phase measurement. In our case, the optical cavity is a microtoroid, whose circumference is modified by mechanical 


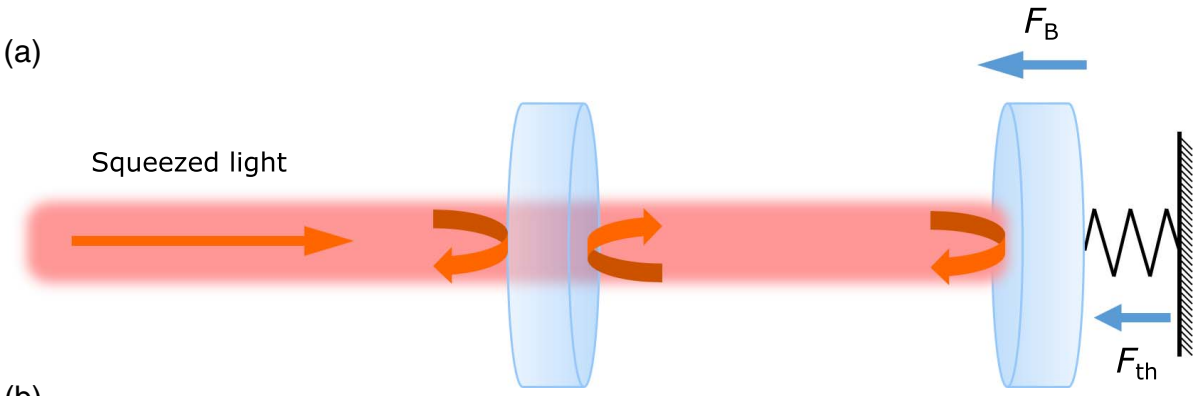

(b)

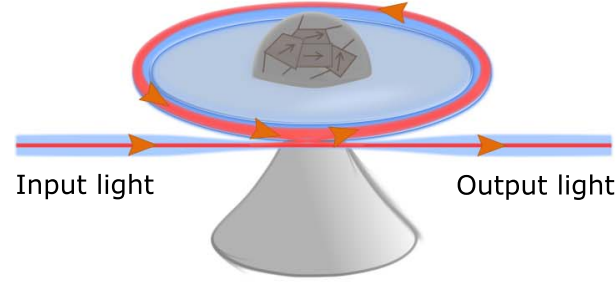

(c)

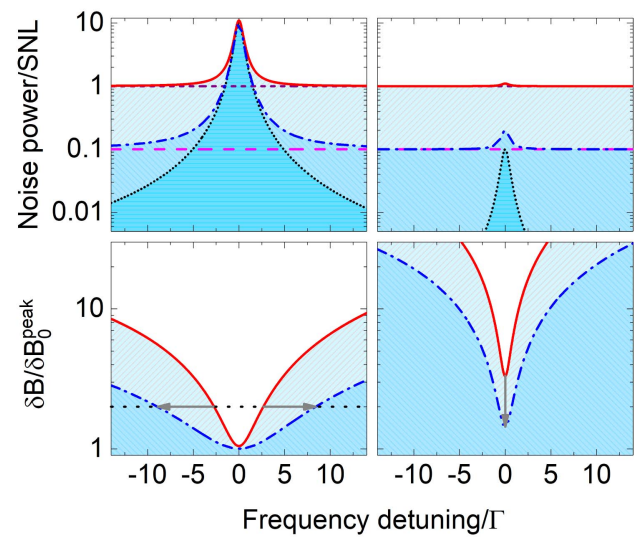

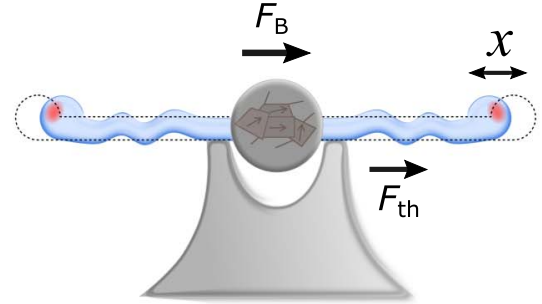

(d)

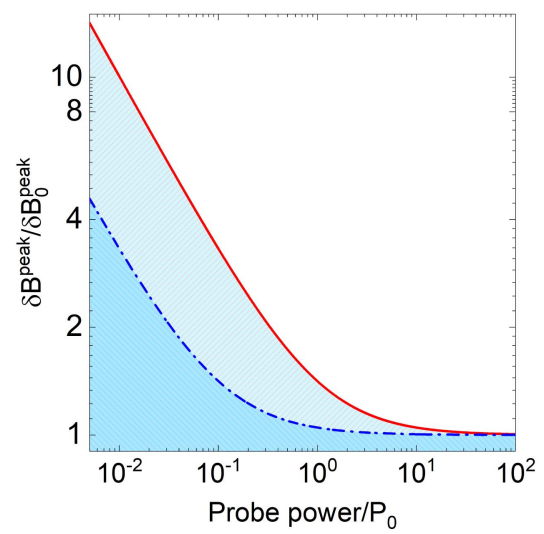

Fig. 1. (a) Conceptual schematic of a cavity optomechanical system probed with squeezed light. Here, $F_{\mathrm{B}}$ and $F_{\text {th }}$ denote the magnetic field induced force and the thermal force on the mechanical oscillator. (b) Left: a schematic of a microtoroid magnetometer coupled with a nanofiber. Right: the cross section of a microtoroid, of which the optical field is distributed along the inner surface. The mechanical motion changes the circumference of the cavity and, thus, shifts the optical resonance. (c) and (d) Theoretical result for squeezing enhanced performance of the magnetometer. Here, we use a squeezing factor of $10 \mathrm{~dB}$. In (c), top-left and bottom-left plots correspond to the strong probe power case, where $P=10 P_{0}$, with $P_{0}$ defined as the power when the thermal noise on mechanical resonance equals the shot noise level (SNL), i.e., $\bar{n}=1 /(16 \eta C)$, while top-right and bottom-right plots correspond to the weak probe power case, where $P=0.1 P_{0}$. (c) Top left and top right, the noise power spectrum normalized to the SNL. Black short-dotted curve, thermal noise; purple short-dashed line, vacuum shot noise for coherent probe; magenta dashed line, squeezed vacuum noise for squeezed probe; red solid curve, total noise for coherent probe; blue dash-dotted curve, total noise for squeezed probe. Bottom left and bottom right, the sensitivity as a function of frequency for coherent (red solid curves) and squeezed (blue dash-dotted curves) probe, respectively, normalized to $\delta B_{0}^{\text {peak }}$, which is the peak sensitivity for the squeezed probe in the strong probe power case. (d) The peak sensitivity $\delta B^{\text {peak }}$ (normalized to $\delta B_{0}^{\text {peak }}$ ) as a function of the probe power $P$ for coherent (red solid curve) and squeezed (blue dash-dotted curve) probes, respectively.

motion, as illustrated in Fig. 1(b). Our experiments operate in the unresolved sideband regime, where the optical decay rate $\kappa$ is much larger than the mechanical resonance frequency $\Omega$. In this regime, the thermal force noise dominates the backaction noise when $\bar{n}>V_{\text {anti }}^{\text {cavity }} C[3]$, where $\bar{n}$ is the thermal phonon occupancy of the mechanical oscillator, $V_{\text {anti }}^{\text {cavity }}$ is the variance of the anti-squeezed field in the optomechanical cavity, and $C$ is the optomechanical cooperativity, which quantifies the strength of radiation pressure optomechanical coupling relative to the mechanical and optical dissipation rates and is proportional to the probe laser power. For the few megahertz frequencies we use, $\bar{n} \sim 10^{6}$ at room temperature; while with the optical and mechanical properties of our optomechanical microresonator, and for the maximum optical power, we use $C \sim 1000$. Consequently, the mechanical force noise is dominated by thermal noise, and we neglect backaction noise henceforth.

The displacement $x$ of the mechanical oscillator in response to an external force $F$ is quantified in the frequency domain by the mechanical susceptibility $\chi(\omega)$. To illustrate the physics, we consider the simple case of a single mechanical resonance, for which $\chi(\omega)=1 /\left(m_{\mathrm{eff}}\left(\Omega^{2}-\omega^{2}-\mathrm{i} \omega \Gamma\right)\right)$, where $m_{\mathrm{eff}}$ is the effective mass of the mechanical oscillator, and $\Gamma$ is its damping rate, enhancing the mechanical response to near resonant forces [see top left and top right of Fig. 1(c)]. Quite generally, in cavity optomechanical sensors, away from resonance, optical shot noise is dominant, allowing squeezed light enhanced sensitivity; while for 
a single-sided cavity in the unresolved sideband regime, thermal noise dominates shot noise at resonance if $\bar{n}>1 /(16 \eta C)$, where $\eta$ is the optical detection efficiency.

A magnetic field is resolvable when the signal it induces is larger than the total noise floor. Neglecting backaction noise, this leads to a minimum detectable force $\delta F$,

$$
\delta F=\sqrt{2 m_{\mathrm{eff}} \Gamma k_{\mathrm{B}} T}\left[1+\frac{V_{\mathrm{sqz}}}{16 \bar{n} \eta C}\left|\frac{\chi(\Omega)}{\chi(\omega)}\right|^{2}\right]^{1 / 2} .
$$

In this equation, $k_{\mathrm{B}}$ and $T$ are the Boltzmann constant and the temperature, respectively. The first term in the bracket on the right hand side represents the thermal noise, while the second term represents the optical noise with $V_{\text {sqz }}$ as the variance of the detected squeezed field. Introducing an actuation constant $c_{\text {act }}=F / B$, which characterizes how well the magnetic field $B$ is converted into an applied force $F$ on the mechanical oscillator [22], the magnetic field sensitivity is $\delta B=\delta F / c_{\text {act }}$.

From Eq. (1), we see that the peak sensitivity occurs on mechanical resonance. In the case where thermal noise is dominant at mechanical resonance frequency $(\bar{n}>1 / 16 C)$, squeezed light does not significantly change the peak sensitivity, instead extending the frequency range over which thermal noise dominates, and, therefore, the sensor bandwidth [bottom left of Fig. 1(c)]; while in the case where optical noise is dominant on resonance $(\bar{n}<1 / 16 C)$, both the peak sensitivity and bandwidth are improved by squeezed light [bottom right of Fig. 1(c)]. The saturation of sensitivity to the optimal (thermal noise limited) sensitivity as probe powers increase is shown in Fig. 1(d). It can be seen that squeezed light reduces the probe power required to reach the optimal sensitivity. We note that similar sensitivity and bandwidth improvements could also be achieved by increasing the probe power. However, this strategy cannot be pursued infinitely due to deleterious effects from the probe, such as detector saturation and absorption heating of the optomechanical device. Of particular relevance to optomechanics, at high probe power, dynamic backaction causes parametric instabilities that degrade sensitivity [43]. These instabilities are especially problematic for the high quality mechanical resonators desirable for precision optomechanical sensing.

\section{EXPERIMENT AND RESULTS}

\section{A. Measurement of the Optomechanical System}

In our experiments, the optomechanical magnetometer is a microtoroid cavity embedded with a grain of magnetostrictive material (terfenol-D) [22,23], as sketched in Fig. 1(b). In such magnetometers, the magnetic field deforms the microcavity via the magnetostrictive expansion and shifts the optical resonance. In the case of an alternating current (AC) magnetic field, the magnetostrictive material exerts a periodic force on the mechanical oscillator, which can drive the mechanical motion of the toroid. When the microcavity is excited on optical resonance, the mechanical motion translates into a pure phase modulation of the transmitted light at the mechanical frequency, which is read out with a homodyne detector and recorded using a spectrum analyzer.

The measurement setup for squeezed light enhanced magnetometry is shown in Fig. 2. A neodymium-doped yttrium aluminum garnet (Nd:YAG) laser is used to produce squeezed light at a wavelength of $1064 \mathrm{~nm}$. Phase-squeezed light is generated through a parametric down conversion process in a $10 \mathrm{~mm}$

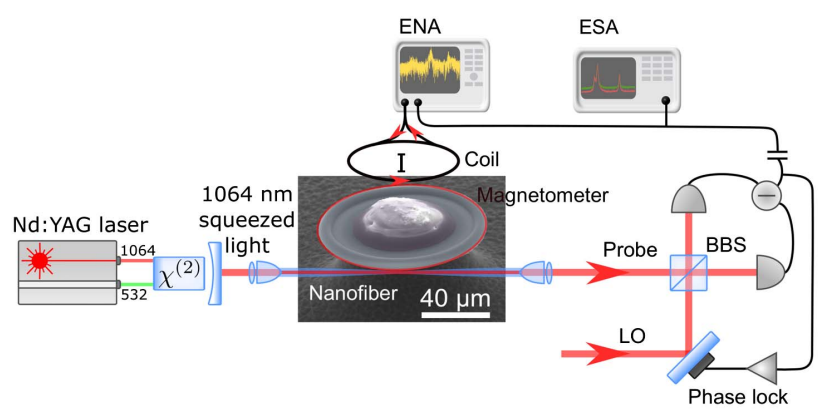

Fig. 2. Measurement setup for squeezed light enhanced cavity optomechanical magnetometry. Squeezed light at a wavelength of $1064 \mathrm{~nm}$ is used to probe the magnetometer. The magnetometer is a microtoroid with terfenol-D embedded inside, as shown in the scanning electron microscope picture. The optical $Q$ factor of the toroid mode is about $1 \times 10^{6}$, corresponding to an optical damping rate of $\kappa / 2 \pi \sim 300 \mathrm{MHz}$. The mechanical motion of the toroid is measured by performing homodyne detection. LO, local oscillator; BBS, balanced beam splitter, comprised of two polarization beam splitters and a half wave plate; ESA, electronic spectrum analyzer; ENA, electronic network analyzer.

periodically poled potassium titanyl phosphate (PPKTP) crystal enclosed in a linear cavity [39]. As shown in Fig. 2, both the $532 \mathrm{~nm}$ light (the pump light) and the $1064 \mathrm{~nm}$ light (the seed light) are injected into the cavity. To generate phase-squeezed light, the pump phase is locked to the seed beam amplification. The light is coupled into the microtoroid evanescently through an optical nanofiber with a diameter of about $700 \mathrm{~nm}$. The optical resonance of the cavity is thermally tuned to match the wavelength of the laser. The cavity phase is actively locked using a feedback system [44]. A coil is used to produce an AC magnetic field to test the magnetic field response of the magnetometer. The mechanical motion of the toroid is measured by performing homodyne detection. The balanced homodyne detector combines two inputs: a relatively weak probe, which couples with the microcavity, and a relatively strong local oscillator (LO), which comes from the same laser but without going through the microcavity. An electronic spectrum analyzer (ESA) is used to record the noise power spectrum. In order to measure the response of the magnetometer to magnetic fields at different frequencies, we drive the coil with the output of an electric network analyzer (ENA) and measure the magnetic field response at each frequency with the same ENA.

\section{B. Characterization of the Squeezed Light}

To characterize the squeezed state transmitted through the fiber, we decouple the microtoroid from the nanofiber and measure the homodyne detection signal of the field quadratures by linearly sweeping the LO phase $\theta$. As shown in the dark gray curve in Fig. 3(a), when $\theta$ is swept continuously, the noise power changes periodically, following the equation $V=V_{\text {sqz }} \cos ^{2} \theta+V_{\text {anti }} \sin ^{2} \theta$, with $V_{\text {anti }}$ being the anti-squeezed quadrature variance. The black solid curve is the fitted result based on this equation, yielding $V_{\text {sqz }}=0.56$ and $V_{\text {anti }}=6.3$. Ideally, the product $V_{\text {sqz }} V_{\text {anti }}=1$, satisfying the Heisenberg uncertainty limit, but, in reality, this limit is not reached, due to loss of the squeezed light during propagation in the setup. The squeezed light source has a squeezing of about $11 \mathrm{~dB}$ (corresponding to a squeezing factor of $V_{\text {sqz }}=0.08$ ), which 
(a)

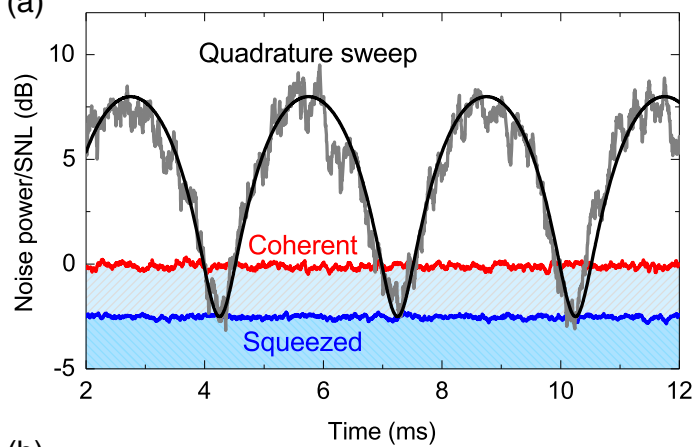

(b)

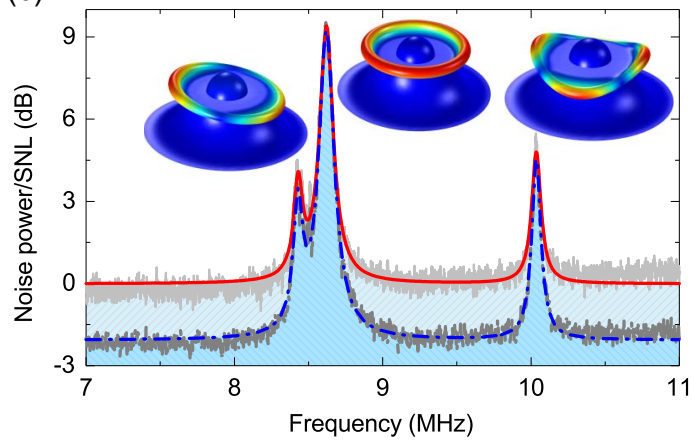

Fig. 3. (a) Characterization of the squeezed state before coupling to the microtoroid. The dark gray curve shows the noise power when sweeping the LO phase continuously with its theoretically fitted result shown in the black solid curve. The red and blue solid curves are the noise power with coherent and squeezed probes when the LO phase is locked at the phase quadrature. (b) The measured noise power from the microtoroid with both coherent (light gray curve) and squeezed (dark gray curve) probes, respectively. The red solid and the blue dashed curves are the fitted results for the measured ones. The three peaks correspond to three mechanical resonance modes (from left to right: tilting mode, flapping mode, and crown mode) with the profiles shown in the inset, obtained using COMSOL Multiphysics.

is degraded by the inefficiencies in the system. These inefficiencies include: loss on the escape from the squeezing cavity, coupling loss from free space into fiber, propagation loss in the nanofiber, coupling loss from free space to the fiber, loss in propagation through optical components, and photodetector inefficiency. Transmission losses of $\sim 37 \%$ through the nanofiber are the dominant source of loss. These losses could be straightforwardly addressed in the future by using a more adiabatic tapered nanofiber, which has been shown to allow transmission losses under $1 \%$ [45]. The overall loss, before coupling to the optomechanical magnetometer, of $54 \%$ degrades $V_{\text {sqz }}$ from 11 to $2.5 \mathrm{~dB}$. The noise power reaches its minimum when locked at the phase quadrature, and we lock $\theta$ to that quadrature henceforth. The red and blue curves in Fig. 3(a) show the noise power for phase quadrature measurement of coherent and squeezed probes, respectively.

\section{Noise Spectra with Coherent and Squeezed Probes}

The squeezed field is coupled into the microcavity through the nanofiber. A trade-off exists when choosing the coupling rate. Critical coupling, where the coupling rate $\kappa_{1}$ equals the intrinsic loss rate $\kappa_{0}$, provides the maximum signal transduction. However, with this choice of coupling, the probe field is entirely absorbed within the cavity, completely removing any squeezing, and, (a)

(b)

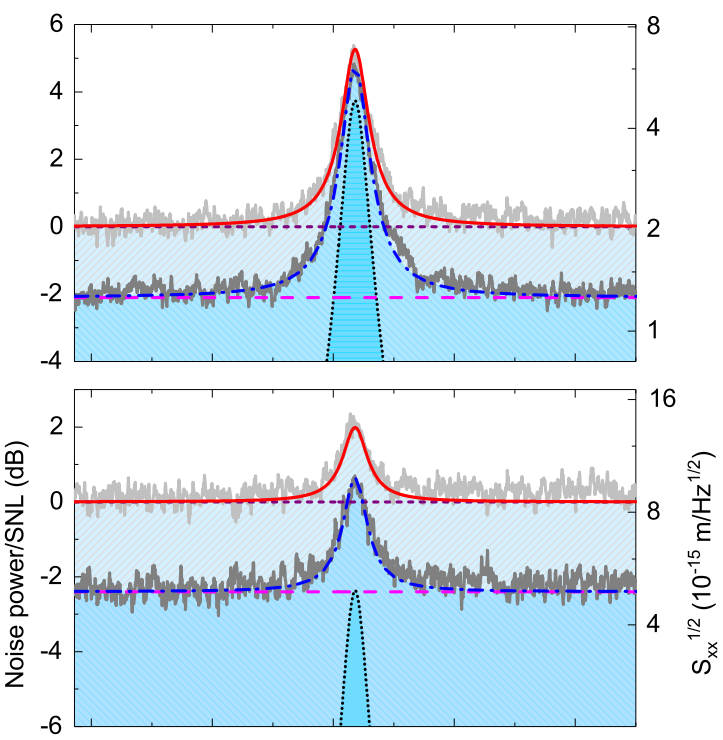

(c)

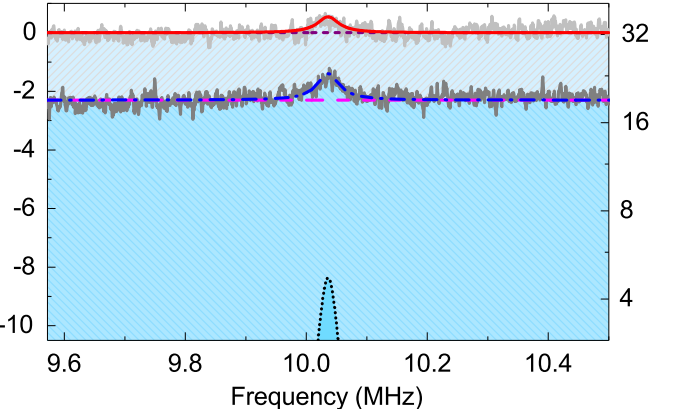

Fig. 4. Characterization of the noise power spectra around the crown mode, under different probe powers: (a) $80 \mu \mathrm{W}$, (b) $20 \mu \mathrm{W}$, and (c) $5 \mu \mathrm{W}$. The light gray and dark gray curves are the measured noise power for coherent and squeezed probes, respectively. The other curves are the theoretically fitted ones: black short-dotted curves, thermal noise; purple short-dashed lines, vacuum shot noise with coherent probe; magenta dashed lines, squeezed vacuum noise with squeezed probe; red solid curves, total noise for the coherent probe; and the blue dash-dotted curves, total noise for the squeezed probe. On the right axes of the figures, it shows the corresponding displacement amplitude spectral density $S_{x x}^{1 / 2}$. The mechanical damping rate is extracted from the linewidth of the mode in the thermal noise spectrum to be $\Gamma / 2 \pi=42 \mathrm{kHz}$. The effective mass of the crown mode is determined to be $m_{\mathrm{eff}}=6.06 \mathrm{ng}$ obtained from COMSOL modeling. The displacement amplitude spectral density $S_{x x}^{1 / 2}$ is plotted on the right axes of the figures.

therefore, prohibiting squeezed light enhancement. Here, we choose a compromise coupling rate $\kappa_{1}=0.52 \kappa_{0}$, which maintains high intracavity power and significant squeezing levels. This coupling rate corresponds to a transmission of about $90 \%$ on optical resonance, and this additional $10 \%$ loss causes a degradation of squeezing from 2.5 to $2.2 \mathrm{~dB}$. The noise power with both coherent and squeezed probes in the frequency range of $7-11 \mathrm{MHz}$ is measured, as shown in the light gray (for coherent probe) and gray (for squeezed probe) curves in Fig. 3(b). With a probe power of $80 \mu \mathrm{W}$, three peaks appear in this frequency range of the noise spectrum, corresponding to three thermally excited mechanical resonance modes. We use COMSOL Multiphysics simulations to identify these three modes as the tilting mode, flapping mode, and crown mode with the corresponding mode profiles shown in the inset. It can be seen that over the frequency 
ranges where the optical noise dominates, the noise floor is suppressed by up to $2.2 \mathrm{~dB}$ by squeezed light, while it is left essentially unchanged when thermal noise dominates.

In order to carefully study the effect of the probe power on the noise spectrum, in the following, we focus on the crown mode with mechanical resonance frequency of $\Omega / 2 \pi=10.035 \mathrm{MHz}$. This mode is chosen due to the particularly clean noise power spectrum in its vicinity. Figure 4(a) shows the noise (normalized to the shot noise level) in the vicinity of the crown mode with probe power $P=80 \mu \mathrm{W}$. As expected, in this case, the noise level remains unchanged by squeezing near the resonance frequency, where thermal noise is dominant and is suppressed away from resonance. As the probe power gradually decreases, the thermal noise drops relative to the shot noise. As shown in Figs. 4(b) and 4(c), the shot noise is dominant in the whole frequency range for probe powers of 20 and $5 \mu \mathrm{W}$. At these power levels, squeezing allows the noise floor to be suppressed over the entire frequency ranges. These results are consistent with the predictions in Fig. 1(c).

\section{Squeezed Light Enhanced Magnetic Field Sensing}

The magnetic field sensitivity of the magnetometer is then characterized. We first characterize the absolute sensitivity at a single frequency $\omega_{\text {ref }} / 2 \pi=8.615 \mathrm{MHz}$. The inset of Fig. 5(a) shows the power spectrum at $\omega_{\text {ref }}$, when the magnetometer is driven with a magnetic field with known strength $B_{\text {ref }}$. The sensitivity at this frequency can be derived from the signal-to-noise ratio (SNR) and $B_{\text {ref }}, \delta B_{\text {ref }}=B_{\text {ref }} / \sqrt{\mathrm{RBW} \times \mathrm{SNR}}$ [22], with RBW being the measurement resolution bandwidth. Figure 5(a) plots the sensitivity at this frequency as a function of the probe power. The red triangles and the blue circles represent the measured result for coherent and squeezed probes, respectively, with the error bars obtained by taking into account the fluctuation of about $\pm 0.5 \mathrm{~dB}$ in the measured noise spectrum. As expected, the sensitivity is improved by squeezing at low probe power, where the shot noise is dominant, and reaches the same optimal sensitivity at high probe power, where the thermal noise is dominant, and in good agreement with theoretical fits. For instance, the sensitivity at $2.5 \mu \mathrm{W}$ probe power is improved from $35.9 \mathrm{nT} / \sqrt{\mathrm{Hz}}$ to $29.2 \mathrm{nT} / \sqrt{\mathrm{Hz}}$, and thermal noise limited sensitivity is about $15.7 \mathrm{nT} / \sqrt{\mathrm{Hz}}$ for both coherent and squeezed probes. The sensitivity at $\omega_{\text {ref }}$ can be used along with the noise spectrum $N_{\omega}$ and network response $R_{\omega}$ to calibrate the sensitivity $\delta B_{\omega}$ over the whole frequency range, $\delta B_{\omega}=\delta B_{\text {ref }} \sqrt{\left(N_{\omega} R_{\text {ref }}\right) /\left(N_{\text {ref }} R_{\omega}\right)}$. This allows the effect of squeezing on bandwidth to be analyzed, as discussed in the following. For a probe power of $80 \mu \mathrm{W}$, the peak sensitivity in the whole frequency range is found to be about $5 \mathrm{nT} / \sqrt{\mathrm{Hz}}$ at $\omega / 2 \pi \sim$ $8.543 \mathrm{MHz}$ for both coherent and squeezed probes.

The sensitivity is found to vary significantly over frequency ranges of around $10 \mathrm{kHz}$ due to resonances in the response of terfenol-D, as shown in the sensitivity spectrum in the bottom-right inset of Fig. 5(b), and consistent with previous observations [22]. This precludes comparison of the magnetometer bandwidth as a function of squeezing to a simple theory. Instead, here, we analyze the squeezing dependence of the accumulated bandwidth, defined as the total frequency range, over which the sensitivity is better than a certain threshold value $\delta B_{\text {thresh }}$ (see bottom-right inset). In Fig. 5(b), we plot the accumulated bandwidth for coherent (red solid curve) and squeezed (blue dash-dotted curve) probes at a probe power of $80 \mu \mathrm{W}$. It can
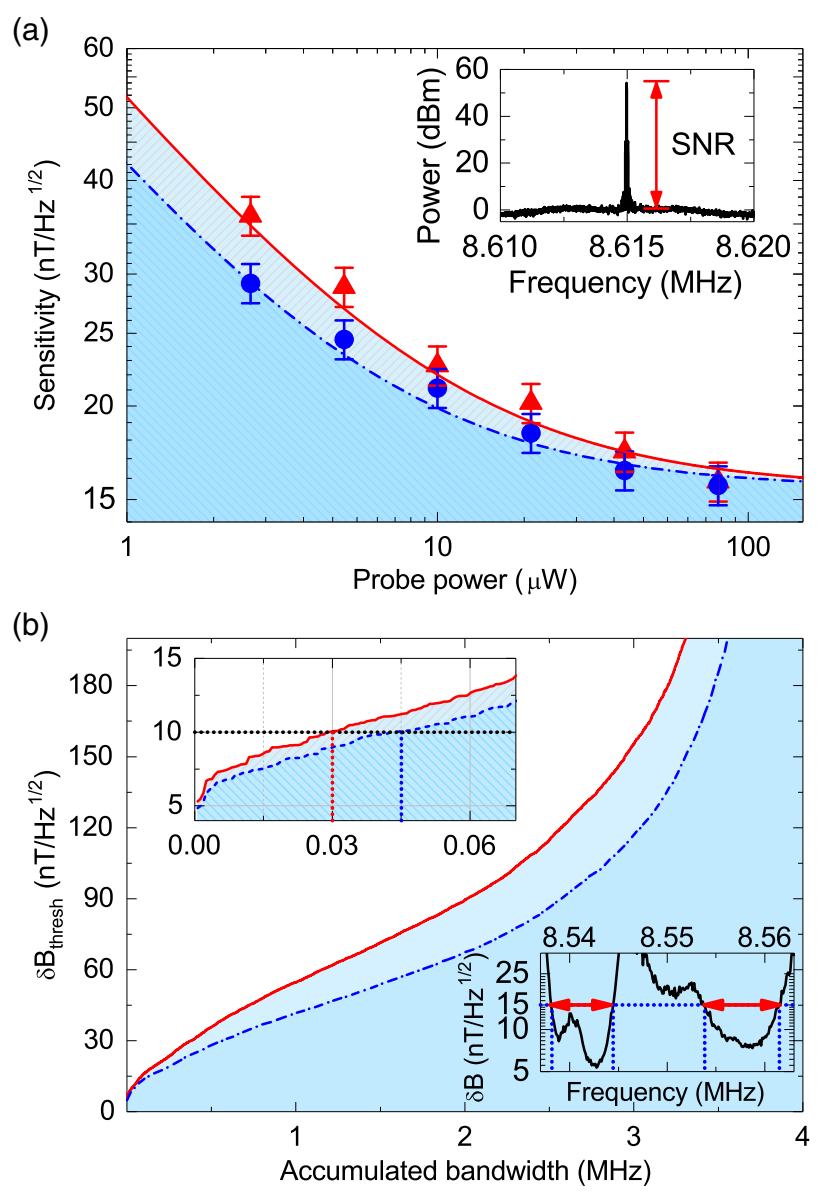

Fig. 5. Sensitivity and bandwidth improvement. (a) Sensitivity at the frequency of $8.615 \mathrm{MHz}$, as a function of the probe power. The red triangles and blue circles represent the measured results for coherent and squeezed probes, respectively. The error bars are obtained by taking into account the fluctuation in the noise power measurement. The red solid (coherent) and blue dash-dotted (squeezed) curves are the corresponding theoretical fitted result. The inset shows the power spectrum when the magnetometer is driven at this frequency with the peak denoting the signal induced by the magnetic field. (b) The accumulated bandwidth as a function of the threshold sensitivity for the coherent (red solid curve) and squeezed (blue dashed curve) probes, respectively. Top-left inset: the zoom-in of the accumulated bandwidth in the frequency range of $0-0.07 \mathrm{MHz}$, showing the $3 \mathrm{~dB}$ bandwidth of $30 \mathrm{kHz}$ for the coherent probe and $45 \mathrm{kHz}$ for the squeezed probe. Bottom-right inset: the sensitivity spectrum in the frequency range of $8.537-8.563 \mathrm{MHz}$, showing the definition of the accumulated bandwidth. For a threshold sensitivity of $15 \mathrm{nT} / \sqrt{\mathrm{Hz}}$, the accumulated bandwidth is defined as the sum of the frequency ranges within the two red arrows.

be seen that, for each $\delta B_{\text {thresh }}$, the accumulated bandwidth for the squeezed probe is greater than that for the coherent probe. The upper-left inset of Fig. 5(b) shows the accumulated bandwidth over the smaller frequency range of $0-70 \mathrm{kHz}$. Squeezed light expands the $3 \mathrm{~dB}$ bandwidth (corresponding to $\delta B_{\text {thresh }}=$ $10 \mathrm{nT} / \sqrt{\mathrm{Hz}}$ ) by $50 \%$, from $30 \mathrm{kHz}$ (for coherent probe) to $45 \mathrm{kHz}$ (for squeezed probe).

\section{CONCLUSIONS}

In summary, we have demonstrated the first application of quantum light in a microcavity optomechanical sensor. By probing a 
cavity optomechanical magnetometer with phase squeezed light, the noise floor is suppressed by about $40 \%$, allowing improved sensitivity by about $20 \%$ in the shot noise dominated regime, and a $50 \%$ enhancement in accumulated bandwidth from 30 to $45 \mathrm{kHz}$. Squeezed light further reduces the optical power required to reach the optimal sensitivity.

Our approach provides a way to improve the sensitivity of the cavity optomechanical magnetometer over a broad frequency range and also opens up possibilities for improving other optomechanical sensors, e.g., inertial sensors [20,21]. While a $20 \%$ improvement in sensitivity is relatively modest; recent advances in squeezing technologies [46-49] hold promise for more substantial improvements. For instance, with detected squeezing of $15 \mathrm{~dB}$ recently reported [49], a sensitivity improvement of a factor of 5.6 could potentially be realized. Moreover, squeezed light could be generated on the same silicon chip as the sensor itself, using radiation pressure induced optomechanical effects $[12,13]$, nonlinear effects in optical resonators [50], or nonlinear waveguides [51]. Further improvements may be possible by optimizing the magnetometer design itself with sensitivities on the order of $100 \mathrm{pT} / \sqrt{\mathrm{Hz}}$ reported in previous cavity optomechanical magnetometers [23]. Sensitivities in this range make cavity optomechanical magnetometers a promising candidate for a range of applications, such as on-chip microfluidic nuclear magnetic resonance for medical diagnosis [52] and magnetoencephalography [53], without the requirement for cryogenic systems that are necessary for other precision magnetometers, such as superconducting quantum interference device (SQUID)-based magnetometers [54,55].

Funding. Villum Fonden (13300); Danmarks Grundforskningsfond (bigQ); Danish Research Council (4184-00338B); Defense Advanced Research Projects Agency (DARPA) (QuASAR program); Australian Research Council (ARC) (DP140100734, FT140100650); Defence Science and Technology Group (DST Group) (CERA49, CERA50); University of Queensland (UQ) (2014001447); National Natural Science Foundation of China (NSFC) (11654003, 61705259).

Acknowledgment. We thank James Bennett, Rachpon Kalra, Christopher Baker, Andreas Sawadsky, Alexander Huck, Jonas Neergaard-Nielsen, and Halina Rubinsztein-Dunlop for the very helpful discussions. W. P. B. acknowledges the Australian Research Future Fellowship. B. B. L. acknowledges the support from the University of Queensland Postdoctoral Research Fellowship and Natural Science Foundation of China. T. G. acknowledges support from the Danish Research Council for Independent Research. Device fabrication was performed within the Queensland Node of the Australian Nanofabrication Facility.

Bei-Bei Li and Jan Bílek contributed equally to this work.

\section{REFERENCES}

1. T. J. Kippenberg and K. J. Vahala, "Cavity optomechanics: back-action at the mesoscale," Science 321, 1172-1176 (2008).

2. M. Aspelmeyer, T. J. Kippenberg, and F. Marquardt, "Cavity optomechanics," Rev. Mod. Phys. 86, 1391-1452 (2014).

3. W. P. Bowen and G. Milburn, Quantum Optomechanics (CRC Press, 2016).
4. A. D. O'Connell, M. Hofheinz, M. Ansmann, R. C. Bialczak, M. Lenander, E. Lucero, M. Neeley, D. Sank, H. Wang, M. Weides, J. Wenner, J. M. Martinis, and A. N. Cleland, "Quantum ground state and single-phonon control of a mechanical resonator," Nature 464, 697-703 (2010).

5. J. Chan, T. P. M. Alegre, A. H. Safavi-Naeini, J. T. Hill, A. Krause, S. Groblacher, M. Aspelmeyer, and O. Painter, "Laser cooling of a nanomechanical oscillator into its quantum ground state," Nature 478, 89-92 (2011).

6. J. D. Teufel, T. Donner, D. Li, J. W. Harlow, M. S. Allman, K. Cicak, A. J. Sirois, J. D. Whittaker, K. W. Lehnert, and R. W. Simmonds, "Sideband cooling of micromechanical motion to the quantum ground state," Nature 475, 359-363 (2011).

7. D. J. Wilson, V. Sudhir, N. Piro, R. Schilling, A. Ghadimi, and T. J. Kippenberg, "Measurement-based control of a mechanical oscillator at its thermal decoherence rate," Nature 524, 325-329 (2015).

8. A. Szorkovszky, A. C. Doherty, G. I. Harris, and W. P. Bowen, "Mechanical squeezing via parametric amplification and weak measurement," Phys. Rev. Lett. 107, 213603 (2011).

9. E. E. Wollman, C. U. Lei, A. J. Weinstein, J. Suh, A. Kronwald, F. Marquardt, A. A. Clerk, and K. C. Schwab, "Quantum squeezing of motion in a mechanical resonator," Science 349, 952-955 (2015).

10. J.-M. Pirkkalainen, E. Damskägg, M. Brandt, F. Massel, and M. Sillanpää, "Squeezing of quantum noise of motion in a micromechanical resonator," Phys. Rev. Lett. 115, 243601 (2015).

11. F. Lecocq, J. Clark, R. Simmonds, J. Aumentado, and J. Teufel, "Quantum nondemolition measurement of a nonclassical state of a massive object," Phys. Rev. X 5, 041037 (2015).

12. A. H. Safavi-Naeini, S. Grblacher, J. T. Hill, J. Chan, M. Aspelmeyer, and O. Painter, "Squeezed light from a silicon micromechanical resonator," Nature 500, 185-189 (2013).

13. T. P. Purdy, P.-L. Yu, R. W. Peterson, N. S. Kampel, and C. A. Regal, "Strong optomechanical squeezing of light," Phys. Rev. X 3, 031012 (2013).

14. A. Schliesser, G. Anetsberger, R. Rivière, O. Arcizet, and T. J. Kippenberg, "High-sensitivity monitoring of micromechanical vibration using optical whispering gallery mode resonators," New J. Phys. 10, 095015 (2008)

15. M. Metcalfe, "Applications of cavity optomechanics," Appl. Phys. Rev. 1, 031105 (2014).

16. A. Abramovici, W. E. Althouse, R. W. P. Drever, Y. Gürsel, S. Kawamura, F. J. Raab, D. Shoemaker, L. Sievers, R. E. Spero, K. S. Thorne, R. E. Vogt, R. Weiss, S. E. Whitcomb, and M. E. Zucker, "LIGO: the laser interferometer gravitational-wave observatory," Science 256, 325-333 (1992).

17. LIGO Scientific Collaboration and Virgo Collaboration, "Observation of gravitational waves from a binary black hole merger," Phys. Rev. Lett. 116, 061102 (2016).

18. J. D. Teufel, T. Donner, M. A. Castellanos-Beltran, J. W. Harlow, and K. W. Lehnert, "Nanomechanical motion measured with an imprecision below that at the standard quantum limit," Nat. Nanotechnol. 4, 820-823 (2009).

19. W. Yu, W. C. Jiang, Q. Lin, and T. Lu, "Cavity optomechanical spring sensing of single molecules," Nat. Commun. 7, 12311 (2016).

20. A. G. Krause, M. Winger, T. D. Blasius, Q. Lin, and O. Painter, "A high resolution microchip optomechanical accelerometer," Nat. Photonics 6 , 768-772 (2012).

21. F. G. Cervantes, L. Kumanchik, J. Pratt, and J. Taylor, "High sensitivity optomechanical reference accelerometer over 10 kHz," Appl. Phys. Lett. 104, 221111 (2014).

22. S. Forstner, S. Prams, J. Knittel, E. D. vanOoijen, J. D. Swaim, G. I. Harris, A. Szorkovszky, W. P. Bowen, and H. Rubinsztein-Dunlop, "Cavity optomechanical magnetometry," Phys. Rev. Lett. 108, 120801 (2012).

23. S. Forstner, E. Sheridan, J. Knittel, C. L. Humphreys, G. A. Brawley, H. Rubinsztein-Dunlop, and W. P. Bowen, "Ultrasensitive optomechanical magnetometry," Adv. Mater. 26, 6348-6353 (2014).

24. C. Yu, J. Janousek, E. Sheridan, D. L. McAuslan, H. Rubinsztein-Dunlop, P. K. Lam, Y. Zhang, and W. P. Bowen, "Optomechanical magnetometry with a macroscopic resonator," Phys. Rev. Appl. 5, 044007 (2016).

25. J. Zhu, G. Zhao, I. Savukov, and L. Yang, "Polymer encapsulated microcavity optomechanical magnetometer," Sci. Rep. 7, 8896 (2017).

26. R. E. Slusher, L. W. Hollberg, B. Yurke, J. C. Mertz, and J. F. Valley, "Observation of squeezed states generated by four-wave mixing in an optical cavity," Phys. Rev. Lett. 55, 2409-2412 (1985). 
27. L.-A. Wu, H. J. Kimble, J. L. Hall, and H. Wu, "Generation of squeezed states by parametric down conversion," Phys. Rev. Lett. 57, 2520-2523 (1986).

28. U. L. Andersen, T. Gehring, C. Marquardt, and G. Leuchs, "30 years of squeezed light generation,” Phys. Scripta 91, 053001 (2016).

29. C. M. Caves, "Quantum-mechanical noise in an interferometer," Phys. Rev. D 23, 1693-1708 (1981).

30. The LIGO Scientific Collaboration, "A gravitational wave observatory operating beyond the quantum shot-noise limit," Nat. Phys. 7, 962-965 (2011).

31. The LIGO Scientific Collaboration, "Enhanced sensitivity of the LIGO gravitational wave detector by using squeezed states of light," Nat. Photonics 7, 613-619 (2013).

32. H. Grote, K. Danzmann, K. L. Dooley, R. Schnabel, J. Slutsky, and H. Vahlbruch, "First long-term application of squeezed states of light in a gravitational-wave observatory," Phys. Rev. Lett. 110, 181101 (2013).

33. M. A. Taylor, J. Janousek, V. Daria, J. Knittel, B. Hage, H. A. Bachor, and W. P. Bowen, "Biological measurement beyond the quantum limit," Nat. Photonics 7, 229-233 (2013).

34. R. C. Pooser and B. Lawie, "Ultrasensitive measurement of microcantilever displacement below the shot-noise limit," Optica 2, 393-399 (2015).

35. N. Treps, N. Grosse, W. P. Bowen, C. Fabre, H.-A. Bachor, and P. K. Lam, "A quantum laser pointer," Science 301, 940-943 (2003).

36. F. Wolfgramm, A. Cere, F. A. Beduini, A. Predojevic, M. Koschorreck, and M. W. Mitchell, "Squeezed-light optical magnetometry," Phys. Rev. Lett. 105, 053601 (2010).

37. T. Horrom, R. Singh, J. P. Dowling, and E. E. Mikhailov, "Quantumenhanced magnetometer with low-frequency squeezing," Phys. Rev. A 86, 023803 (2012).

38. U. B. Hoff, G. I. Harris, L. S. Madsen, H. Kerdoncuff, M. Lassen, B. M. Nielsen, W. P. Bowen, and U. L. Andersen, "Quantum-enhanced micromechanical displacement sensitivity," Opt. Lett. 38, 1413-1415 (2013).

39. C. Schäfermeier, H. Kerdoncuff, U. B. Hoff, H. Fu, A. Huck, J. Bilek, G. I. Harris, W. P. Bowen, T. Gehring, and U. L. Andersen, "Quantum enhanced feedback cooling of a mechanical oscillator using nonclassical light," Nat. Commun. 7, 13628 (2016).

40. J. B. Clark, F. Lecocq, R. W. Simmonds, J. Aumentado, and J. D. Teufel, "Sideband cooling beyond the quantum backaction limit with squeezed light," Nature 541, 191-195 (2017).

41. J. B. Clark, F. Lecocq, R. W. Simmonds, J. Aumentado, and J. D. Teufel, "Observation of strong radiation pressure forces from squeezed light on a mechanical oscillator," Nat. Phys. 12, 683-687 (2016).

42. M. Korobko, L. Kleybolte, S. Ast, H. Miao, Y. Chen, and R. Schnabel, "Beating the standard sensitivity-bandwidth limit of cavity-enhanced interferometers with internal squeezed-light generation," Phys. Rev. Lett. 118, 143601 (2017).

43. G. I. Harris, U. L. Andersen, J. Knittel, and W. P. Bowen, "Feedbackenhanced sensitivity in optomechanics: surpassing the parametric instability barrier," Phys. Rev. A 85, 061802 (2012).

44. E. D. Black, "An introduction to Pound-Drever-Hall laser frequency stabilization," Am. J. Phys. 69, 79-87 (2001).

45. L. Ding, C. Belacel, S. Ducci, G. Leo, and I. Favero, "Ultralow loss singlemode silica tapers manufactured by a microheater," Appl. Opt. 49, 24412445 (2010).

46. E. S. Polzik, J. Carri, and H. J. Kimble, "Atomic spectroscopy with squeezed light for sensitivity beyond the vacuum-state limit," Appl. Phys. B 55, 279-290 (1992).

47. Y. Takeno, M. Yukawa, H. Yonezawa, and A. Furusawa, "Observation of -9 dB quadrature squeezing with improvement of phase stability in homodyne measurement," Opt. Express 15, 4321-4327 (2007).

48. T. Eberle, S. Steinlechner, J. Bauchrowitz, V. Handchen, H. Vahlbruch, M. Mehmet, H. Müller-Ebhardt, and R. Schnabel, "Quantum enhancement of the zero-area sagnac interferometer topology for gravitational wave detection," Phys. Rev. Lett. 104, 251102 (2010).

49. H. Vahlbruch, M. Mehmet, K. Danzmann, and R. Schnabel, "Detection of $15 \mathrm{~dB}$ squeezed states of light and their application for the absolute calibration of photoelectric quantum efficiency," Phys. Rev. Lett. 117, 110801 (2016).

50. A. Dutt, K. Luke, S. Manipatruni, A. L. Gaeta, P. Nussenzveig, and M. Lipson, "On-chip optical squeezing," Phys. Rev. Appl. 3, 044005 (2015).

51. U. B. Hoff, B. M. Nielsen, and U. L. Andersen, "Integrated source of broadband quadrature squeezed light," Opt. Express 23, 1201312036 (2015).

52. I. Savukov and T. Karaulanov, "Magnetic-resonance imaging of the human brain with an atomic magnetometer," Appl. Phys. Lett. 103, 043703 (2013).

53. H. Xia, A. Ben-Amar Baranga, D. Hoffman, and M. V. Romalis, "Magnetoencephalography with an atomic magnetometer," Appl. Phys. Lett. 89, 211104 (2006).

54. J. R. Kirtley, M. B. Ketchen, K. G. Stawiasz, J. Z. Sun, W. J. Gallagher, S. H. Blanton, and S. J. Wind, "High-resolution scanning SQUID microscope," Appl. Phys. Lett. 66, 1138-1140 (1995).

55. F. Baudenbacher, L. E. Fong, J. R. Holzer, and M. Radparvar, "Monolithic low-transition-temperature superconducting magnetometers for high resolution imaging magnetic fields of room temperature samples," Appl. Phys. Lett. 82, 3487-3489 (2003). 